\title{
Parton Distributions with the Combined HERA Charm Production Cross Sections
}

\author{
Valerio Bertone* and Juan Rojo ${ }^{\dagger}$ \\ * Physikalisches Institut, Albert-Ludwigs-Universität Freiburg, \\ Hermann-Herder-Straße 3, D-79104 Freiburg i. B., Germany. \\ ${ }_{\dagger}$ PH Department, TH Unit, CERN, CH-1211 Geneva 23, Switzerland. \\ Results presented on behalf of the NNPDF Collaboration, ht tp://nnpdf. hepforge.org/
}

\begin{abstract}
Heavy quark structure functions from HERA provide a direct handle on the medium and small- $x$ gluon PDF. In this contribution, we discuss ongoing progress on the implementation of the FONLL General-Mass scheme with running heavy quark masses, and of its benchmarking with the HOPPET and OpenQCDrad codes, and then present the impact of the recently released combined HERA charm production cross sections in the NNPDF2.3 analysis. We find that the combined charm data contribute to constraining the gluon and quarks at small values of Bjorken- $x$.
\end{abstract}

Keywords: Parton Distributions, Heavy Quarks, Charm Production

PACS: 14.65.Dw, 12.38.-t,

Charm structure function data and PDF fits. Charm production in deep-inelastic scattering is directly sensitive to the gluon PDF. The ZEUS and H1 collaborations at the HERA collider have measured charm production in DIS with a wide variety of techniques, and these $F_{2}^{c}$ data is included in all modern PDF fits (see [1] for a recent overview). The HERA experiments have recently released their combined data on charm production cross sections from HERA Runs I and II [2], where a common consistent data set with the full correlation matrix is provided, and in addition the combination procedure yields systematic errors rather smaller than what one would expect from the naive combination of all the data, because of the mutual cross-calibration between $\mathrm{H} 1$ and ZEUS.

In this contribution we explore the impact of replacing the separated H1 and ZEUS $F_{2}^{c}$ data with the combined charm production cross sections $\tilde{\sigma}_{\mathrm{NC}}^{c}$ in the NNPDF2.3 analysis [3]. We also discuss how one can generalize the FONLL General-Mass VFN scheme to include running heavy quark masses in the $\overline{\mathrm{MS}}$ scheme. Heavy quark structure functions in the $\overline{\mathrm{MS}}$ scheme lead to a better behaved perturbative expansion than pole masses [4] and allow to compare the value of the heavy quark masses used with those determined by other experiments (e. g. by LEP data).

FONLL Structure functions in the $\overline{M S}$ scheme. Treating heavy quark structure functions in the $\overline{\mathrm{MS}}$ scheme results in an improved convergence of the perturbative expansion and allows to consistently compare the mass of the charm quark used in the PDF analysis with other determinations. The NNPDF fits are based on the FONLL GM-VFN scheme for heavy quark structure functions [5], with pole masses as default. It can be shown that FONLL can be extended to use $\overline{\mathrm{MS}}$ heavy quark masses, we discuss here some progress 
TABLE 1. Relative differences at $Q^{2}=10^{4} \mathrm{GeV}^{2}$ for NNLO PDF evolution in the massless scheme with $\overline{\mathrm{MS}}$ running heavy quark masses as implemented in the FastKernel framework in comparison to HOPPET, $\varepsilon_{\text {rel }} \equiv\left|\left(q_{i}^{\mathrm{fk}}\left(x, Q^{2}\right)-q_{i}^{\mathrm{hop}}\left(x, Q^{2}\right)\right) / q_{i}^{\mathrm{fk}}\left(x, Q^{2}\right)\right|$, for various PDF flavor combinations. The PDFs and the settings of the comparison are the same as in the Les Houches benchmark comparisons [7].

\begin{tabular}{|c||c|c|c|c|c|}
\hline$x$ & $\varepsilon_{\text {rel }}\left(u_{v}\right)$ & $\varepsilon_{\text {rel }}\left(d_{v}\right)$ & $\varepsilon_{\text {rel }}\left(L_{+}\right)$ & $\varepsilon_{\text {rel }}\left(c^{+}\right)$ & $\varepsilon_{\text {rel }}(g)$ \\
\hline \hline $1.0 \cdot 10^{-5}$ & $2.30 \cdot 10^{-4}$ & $2.63 \cdot 10^{-4}$ & $3.28 \cdot 10^{-5}$ & $7.10 \cdot 10^{-5}$ & $9.39 \cdot 10^{-5}$ \\
$1.0 \cdot 10^{-3}$ & $1.23 \cdot 10^{-4}$ & $9.18 \cdot 10^{-5}$ & $6.77 \cdot 10^{-5}$ & $8.86 \cdot 10^{-5}$ & $1.02 \cdot 10^{-4}$ \\
$1.0 \cdot 10^{-2}$ & $2.63 \cdot 10^{-4}$ & $3.12 \cdot 10^{-4}$ & $9.06 \cdot 10^{-5}$ & $1.59 \cdot 10^{-4}$ & $1.30 \cdot 10^{-4}$ \\
$1.0 \cdot 10^{-1}$ & $2.69 \cdot 10^{-4}$ & $3.99 \cdot 10^{-4}$ & $5.29 \cdot 10^{-4}$ & $3.36 \cdot 10^{-5}$ & $9.15 \cdot 10^{-5}$ \\
$3.0 \cdot 10^{-1}$ & $2.77 \cdot 10^{-5}$ & $2.77 \cdot 10^{-5}$ & $3.79 \cdot 10^{-4}$ & $3.79 \cdot 10^{-4}$ & $7.21 \cdot 10^{-5}$ \\
$7.0 \cdot 10^{-1}$ & $1.87 \cdot 10^{-4}$ & $1.21 \cdot 10^{-4}$ & $1.56 \cdot 10^{-3}$ & $3.75 \cdot 10^{-2}$ & $1.44 \cdot 10^{-3}$ \\
\hline
\end{tabular}

in the this direction. Let us recall that FONLL can combine different perturbative orders for massive and massless structure functions: FONLL-A combines the NLO massless with $\mathscr{O}\left(\alpha_{s}\right)$ massive pieces, FONLL-C achieves the same at $\mathscr{O}\left(\alpha_{s}^{2}\right)$ and FONLL-B is an intermediate case where the $\mathscr{O}\left(\alpha_{s}^{2}\right)$ massive calculation is combined with the NLO massless result.

The first ingredient to consider, as compared to pole masses, is the NNLO massless PDF evolution, which is modified by the scheme transformation between pole and $\overline{\mathrm{MS}}$ running masses (NLO evolution is identical in the two schemes). In particular, the heavy quark thresholds require different matching conditions with running masses as compared to pole masses [4]. To benchmark the implementation of the $\overline{\mathrm{MS}}$ NNLO PDF evolution in the NNPDF FastKernel framework, we report in Table 1 the relative accuracy against the $x$-space evolution code HOPPET [6], that also has the option to perform PDF evolution with running heavy quark masses. This benchmark has been performed using the same parameters as in the Les Houches PDF comparison [7]. We find excellent agreement over all the kinematic range.

A second ingredient of the FONLL $\overline{\mathrm{MS}}$ masses implementation is the comparison with the OpenQCDrad code ${ }^{1}$, which provides charm structure functions in the $N_{f}=3$ FFN scheme both for pole masses and for $\overline{\mathrm{MS}}$ running masses. The comparison is done as follows. First of all, we compute charm structure functions in the massive scheme with pole masses set to $M_{c}=\sqrt{2} \mathrm{GeV}$ in both codes, and check that there is reasonable agreement. Then we transform the pole mass to the $\overline{\mathrm{MS}}$ running mass, which is this case corresponds to $m_{c}\left(m_{c}\right)=1.06 \mathrm{GeV}$, and use this as input of the $\overline{\mathrm{MS}}$ running mass computation both in FastKernel in the FFN scheme and in OpenQCDrad. In Table 2 we present the percentage difference for charm structure function $F_{2}^{c}$ in the $\overline{\mathrm{MS}}$ scheme, we find good agreement over a wide kinematical range.

With these two ingredients, it is possible to generalize FONLL to include running

1 http: / / www-zeuthen.desy.de/ alekhin/OPENQCDRAD / 
TABLE 2. Relative differences between the FastKernel implementation of the charm structure function $F_{2}^{c}$ with $\overline{\mathrm{MS}}$ heavy quark running masses and the OpenQCDrad results, $\varepsilon_{\text {rel }} \equiv\left|\left(F_{2 c}^{\mathrm{fk}}\left(x, Q^{2}\right)-F_{2 c}^{\mathrm{oqr}}\left(x, Q^{2}\right)\right) / F_{2 c}^{\mathrm{fk}}\left(x, Q^{2}\right)\right|$. The computation has been performed in the massive scheme at order $\mathscr{O}\left(\alpha_{s}^{2}\right)$ with the LH toy PDFs.

\begin{tabular}{|c|c|c|c|}
\hline$x \backslash Q^{2}$ & $\varepsilon_{\text {rel }}\left(Q^{2}=10 \mathrm{GeV}^{2}\right)$ & $\varepsilon_{\text {rel }}\left(Q^{2}=100 \mathrm{GeV}^{2}\right)$ & $\varepsilon_{\mathrm{rel}}\left(Q^{2}=1000 \mathrm{GeV}^{2}\right)$ \\
\hline $10^{-4}$ & $1.8 \%$ & $1.8 \%$ & $1.1 \%$ \\
\hline $10^{-3}$ & $0.1 \%$ & $0.1 \%$ & $0.4 \%$ \\
\hline $10^{-2}$ & $0.2 \%$ & $0.1 \%$ & $0.8 \%$ \\
\hline
\end{tabular}
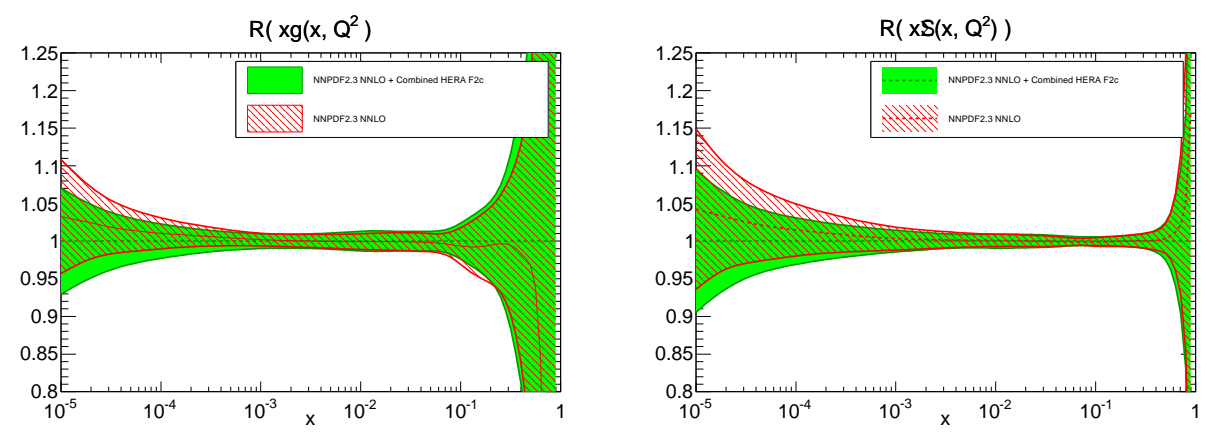

FIGURE 1. The ratio of the gluon (left plot) and quark singlet (right plot) between the NNPDF2.3 PDFs and the fit where with the separated $F_{2}^{c} \mathrm{H} 1$ and ZEUS data have been replaced by the combined $\widetilde{\sigma}_{\mathrm{NC}}^{c}$ charm reduced cross sections. PDFs have been evaluated at a typical LHC scale of $Q^{2}=10^{4} \mathrm{GeV}^{2}$.

heavy quark masses. Of course the difference between pole and running masses appears only at $\mathscr{O}\left(\alpha_{s}^{2}\right)$, so FONLL-A will not be changed, while both FONLL-B and FONLL$\mathrm{C}$ will be modified by the scheme transformation. A more detailed discussion of the implementation of running heavy quark masses in FONLL, and the corresponding impact on parton distributions, will be presented elsewhere.

The impact of the combined HERA charm production data. The NNPDF analysis have included all the available $\mathrm{H} 1$ and ZEUS charm structure function data since NNPDF2.1 [8, 9, 10, 3]. We have now implemented the combined HERA charm production cross sections $\widetilde{\sigma}_{\mathrm{NC}}^{c}$ in the NNPDF code, and performed various fits, at NLO and NNLO, with the same settings as NNPDF2.3 but replacing the separated H1 and ZEUS $F_{2}^{c}$ data with the combined HERA data. We take fully into account the 43 sources of correlated systematics of the combined dataset, which include normalization and procedural uncertainties. To ease the comparison, structure functions are computed in the pole mass scheme with the same mass values as in NNPDF2.3. We obtain a good description of the data, with a $\chi^{2}$ per data point of about 1.2.

We show the impact of the combined HERA charm data on NNPDF2.3 NNLO in Fig. 1 and we also show the associated distances between the two fits in Fig. 2. As we can see, the net effect of replacing the separated $F_{2}^{c}$ data with the combined reduced cross 

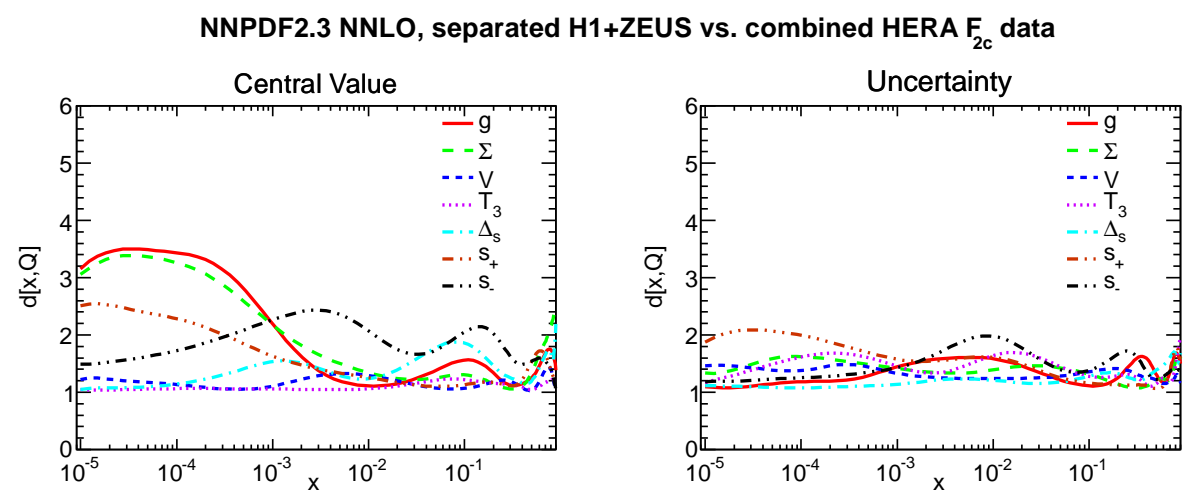

FIGURE 2. Distances between the two fits of Fig. 1. See [11] for the definitions.

sections is to shift the central value of the small- $x$ gluon and total quark singlet PDFs by a moderate amount, between one third and half a sigma of the PDF uncertainty.

Outlook. We have discussed the implementation of the combined HERA charm data in the NNPDF framework, showed that these new data provide some useful constrains on the poorly known small- $x$ gluons and quarks, and reported on the implementation of the FONLL GM scheme with running heavy quark masses. We plan to use these results to perform a determination of $m_{c}\left(m_{c}\right)$ from the combined HERA charm production data, with the same techniques used to determine $\alpha_{s}\left(M_{Z}\right)[12,13]$.

\section{ACKNOWLEDGMENTS}

We thank M. Corradi and K. Lipka for assistance with the $F_{2}^{c}$ HERA data, and G. Salam for discussions on HOPPET. J. R. is supported by a Marie Curie Intra-European Fellowship of the European Community's 7th Framework Programme under contract number PIEF-GA-2010-272515.

\section{REFERENCES}

1. R. D. Ball, S. Carrazza, L. Del Debbio, S. Forte, et al. (2012), 1211.5142.

2. H. Abramowicz, et al. (2012), 1211.1182.

3. R. D. Ball, V. Bertone, S. Carrazza, et al., Nucl.Phys. B867, 244-289 (2013), 1207.1303.

4. S. Alekhin, and S. Moch, Phys.Lett. B699, 345-353 (2011), 1011.5790.

5. S. Forte, E. Laenen, P. Nason, and J. Rojo, Nucl. Phys. B834, 116-162 (2010), 1001.2312.

6. G. P. Salam, and J. Rojo, Comput. Phys. Commun. 180, 120-156 (2009), 0804.3755.

7. M. Dittmar, et al. (2005), hep-ph/0511119.

8. R. D. Ball, et al., Nucl. Phys. B849, 296-363 (2011), 1101.1300.

9. R. D. Ball, et al., Nucl.Phys. B855, 153-221 (2012), 1107 . 2652.

10. R. D. Ball, V. Bertone, F. Cerutti, L. Del Debbio, S. Forte, et al., Nucl.Phys. B855, 608-638 (2012), 1108.1758.

11. R. D. Ball, et al., Nucl. Phys. B838, 136-206 (2010), 1002.4407.

12. S. Lionetti, et al., Phys. Lett. B701, 346-352 (2011), 1103.2369.

13. R. D. Ball, V. Bertone, L. Del Debbio, et al., Phys.Lett. B707, 66-71 (2012), 1110 . 2483. 